\title{
Effect of fuel composition and differential diffusion on flame stabilization in reacting syngas jets in turbulent cross-flow
}

\author{
Yuki Minamoto $^{1}$, Hemanth Kolla ${ }^{1}$, Ray W. Grout ${ }^{2}$, Andrea Gruber ${ }^{3}$, \\ Jacqueline H. Chen ${ }^{1}$ \\ ${ }^{1}$ Sandia National Laboratories, Livermore, CA 94550, USA \\ ${ }^{2}$ National Renewable Energy Laboratory, Golden, CO 80401, USA \\ ${ }^{3}$ SINTEF Energy Research, K. Heiesvei 1A, 7465 Trondheim, Norway
}

\begin{abstract}
Three-dimensional direct numerical simulation results of a transverse syngas fuel jet in turbulent cross-flow of air are analyzed to study the influence of varying volume fractions of $\mathrm{CO}$ relative to $\mathrm{H}_{2}$ in the fuel composition on the near field flame stabilization. The mean flame stabilizes at a similar location for CO-lean and CO-rich cases despite the trend suggested by their laminar flame speed, which is higher for the CO-lean condition. To identify local mixtures having favorable mixture conditions for flame stabilization, explosive zones are defined using a chemical explosive mode timescale. The explosive zones related to flame stabilization are located in relatively low velocity regions. The explosive zones are characterized by excess hydrogen transported solely by differential diffusion, in the absence of intense turbulent mixing or scalar dissipation rate. The conditional averages show that differential diffusion is negatively correlated with turbulent mixing. Moreover, the local turbulent Reynolds number is insufficient to estimate the magnitude of the differential diffusion effect. Alternatively, the Karlovitz number provides a better indicator of the importance of differential diffusion. A comparison of the variations of differential diffusion, turbulent mixing, heat release rate and probability of encountering explosive zones demonstrates that differential diffusion predominantly plays an important role for mixture preparation and initiation of chemical reactions, closely followed by intense chemical reactions sustained by sufficient downstream turbulent mixing. The mechanism
\end{abstract}


by which differential diffusion contributes to mixture preparation is investigated using the Takeno Flame Index. The mean Flame Index, based on the combined fuel species, shows that the overall extent of premixing is not intense in the upstream regions. However, the Flame Index computed based on individual contribution of $\mathrm{H}_{2}$ or $\mathrm{CO}$ species reveals that hydrogen contributes significantly to premixing, particularly in explosive zones in the upstream leeward region, i.e. the preferred flame stabilization location. Therefore, a small amount of $\mathrm{H}_{2}$ diffuses much faster than $\mathrm{CO}$, creating relatively homogeneous mixture pockets depending on the competition with turbulent mixing. These pockets, together with high $\mathrm{H}_{2}$ reactivity, contribute to stabilizing the flame at a consistent location regardless of the $\mathrm{CO}$ concentration in the fuel for the present range of DNS conditions.

Keywords: Flame stabilization, Transverse jet, Syngas combustion, Direct numerical simulation

\section{Introduction}

The Integrated Gasification Combined Cycle (IGCC) represents a potential route to a more efficient and environmental-friendly power generation of solid fuels (coal and/or bio-mass). One reason for this is that in the IGCC a synthetic gaseous fuel (syngas) derived from the solid fuel can be burned in a gas and steam turbine combined cycle, which has a higher thermal efficiency than a steam power plant operating with pulverized coal combustion (PCC). Another advantage, in the context of power generation with $\mathrm{CO}_{2}$ capture, is that after the Water-Gas Shift (WGS) reaction, the high pressure and $\mathrm{CO}_{2}$ concentration of the $\mathrm{H}_{2}$-rich syngas fuel enables an energy-efficient $\mathrm{CO} 2$ capture process. After an initial phase in which the complexity and cost of the IGCC process led to a slow and intermittent deployment of IGCC power plants, interest in the technology has been renewed recently with plants being commissioned in China, Japan and the US [1].

Several problems of practical importance must be addressed in the development of a combustion system capable of efficient, clean and safe combustion of IGCC syngas: in particular, the often varying composition of the fuel, that can contain large fractions of hydrogen, must be taken into account at the combustor design stage, especially if the system must comply with the emissions regulations for threshold levels of $\mathrm{CO}$ and NOx. Some of these problems are well understood and utilize well-developed engineering 
knowledge. However, there are a number of other issues involving complex interactions between the flow field and the reaction kinetics associated with the fundamental combustion process that are far less understood [2]. More specifically, burners are often designed on the basis of complex, poorly understood balances between the flow field, the process of fuel-oxidant mixing and flame propagation. One of the most serious operability issues often preventing modern combustors from operating in a safe, efficient, and reliable manner is flashback when the flame propagates upstream of the design position, where it is supposed to stabilize, and into the premixing duct [3]. Common flashback mechanisms include propagation of the turbulent flame in the core flow of the premixer duct or in its wall boundary layers, oscillations of the flame location due to combustion instabilities or combustion induced vortex breakdown [5]. Regardless of the initiating cause of flashback, once flashback occurs the flame will ultimately reach the fuel injection nozzles. Therefore, understanding and accurate prediction of the flame stabilization mechanism in the near field of fuel jet in cross flow of oxidant are vitally important for gas turbine combustor design. More specifically to applications involving premixed combustion of hydrogen-rich syngas at gas turbine conditions (high pressure, high reactant temperature) mounting evidence indicates that boundary layer flashback constitutes a key challenge [5]. Boundary layer flashback can result in high speed upstream flame propagation, even in the presence of large flow velocities in the downstream direction for the reactants in the bulk region of the duct. This has been shown recently in both experimental and modelling studies $[6,7,8]$. Once flashback occurs, it can lead to flame anchoring in the near field of the fuel injection nozzles, rapid increase of material temperatures, with subsequent damage and failure [2]. In order to ensure intrinsic flashback safety, flame stabilization in the near field of the fuel injection nozzles needs to be understood.

A transverse jet in cross-flow (JICF) configuration is often used to achieve a high level of fuel-air mixing within a confined volume and short residence time, and hence it is used in many engineering applications including gas turbines in IGCC. However, the JICF generates a complex three-dimensional flow field involving a variety of flow structures spanning a broad range of length and time scales: shear layer vortices, wake vortices, horse-shoe vortices, and a counter-rotating vortex pair (CVP) [9]. While these flow structures are pivotal in enhancing mixing and in affecting flame stabilization characteristics, they are challenging to study and characterize due to their strong three-dimensionality. Passive scalar transport in the JICF has been stud- 
ied experimentally [10] and numerically [11], and these studies have shown that scalar mixing in JICF might not be modeled through a direct analogue with the turbulent flow field. In a reacting JICF heat release due to chemical reactions coupled with organized turbulent motion influence the scalar transport in a manner different than passive scalar transport. Also, preferential diffusion of species, with mass diffusivities varying by as much as five-fold, can modify the scalar mixing characteristics $[12,13]$. Therefore, flame stabilization depends upon the balance of these transport phenomena.

The issue of flame anchoring and stabilization in the near field of a fuel injection nozzle in JICF was previously studied by Direct Numerical Simulations (DNS). Several parameters that influence the JICF near field flame stabilization were investigated. These parameters include fuel nozzle geometry $[14,15]$ and injection angle with respect to the cross flow direction [16]. Previous experimental $[17,18]$ and DNS $[14,15]$ studies have reported that a flame stabilizes in the near-field of a JICF via partially premixed flame propagation. Indeed, the flow velocity component normal to the mean flame base, measured conditionally at the instantaneous flame base location, was reported to be strongly correlated to the laminar flame speed [17]. In light of this, the fuel composition may be expected to have a dominant influence on flame stabilization since it considerably alters the laminar flame propagation speed and flame thickness. This is particularly true of syngas used in IGCC since its two components - hydrogen $\left(\mathrm{H}_{2}\right)$ and carbon monoxide $(\mathrm{CO})$ - have widely disparate chemical and transport properties. Well resolved DNS with detailed chemical kinetics and transport properties can greatly illuminate the parametric influence of fuel composition.

The present study is a continuation of a series of numerical parametric studies $[14,15,16]$ to understand key sensitivities in the JICF near field stabilization of multi-component fuel combustion. While previous studies varied fuel nozzle geometry [14, 15] and transverse injection angle [16], in the present study the focus is on the effect of composition of hydrogen-rich syngas fuel on flame stabilization characteristics relevant to IGCC. Thus, the present study augments the existing parametric studies. Here, we quantify the relative importance of differential diffusion, turbulent mixing and turbulence-chemistry interaction in achieving flame anchoring. More specifically, the Chemical Explosive Mode Analysis (see Section 3) is used in combination with analysis of the TAKENO flame/mixing index.

The remainder of this paper is organized as follows: the numerical methods in the DNS code, S3D, used to perform the present simulations is de- 
scribed in Sec. 2, followed by a detailed description of the DNS configuration and numerical and thermochemical conditions. Instantaneous and time-averaged results from the reacting DNS are discussed in Sec. 3. Finally, conclusions and recommendations for further work are presented in Sec. 4.

\section{Direct numerical simulations}

The Sandia DNS code S3D [19] is used to simulate the combustion of two $\mathrm{H}_{2} / \mathrm{CO}$-air flames aerodynamically stabilized in the near field of the transverse jet immediately downstream of the fuel nozzle. The code solves fully-compressible conservation equations for mass, momentum, total energy and species continuity. The chemical reactions are described by a skeletal mechanism for $\mathrm{H}_{2} / \mathrm{CO}$-air combustion involving $N=12$ species and 29 elementary reactions [20]. Nitrogen is inert in this context and therefore, $\mathrm{NO}_{x}$ formation reactions are not included. The species specific heats are modeled as polynomial functions of temperature as described in CHEMKIN and TRANSPORT [21], and mixture-averaged transport coefficients are used [22]. Radiative heat transfer is not considered in the present simulations. Spatial derivatives are obtained using an eighth-order central finite difference scheme which gradually reduces to a third-order one-sided difference stencil on the open boundaries [23]. A tenth-order explicit spatial filter is applied to remove any spurious high-frequency fluctuation in the solution [23]. Time integration is achieved using a six-stage fourth-order explicit Runge-Kutta method [23].

The numerical configuration is shown in Fig. 1. The computational domain is a cuboid with dimensions, $L_{x} \times L_{y} \times L_{z}=25 \times 20 \times 20 \mathrm{~mm}^{3}$, respectively. The Navier-Stokes Characteristic Boundary Conditions (NSCBC) are implemented $[24,25]$ for non-reflecting inflow $(x=0)$ and outflow $\left(x=L_{x}\right.$ and $\left.y=L_{y}\right)$ planes and a no-slip isothermal, solid surface wall $(y=0)$ boundaries while the $z$ boundaries are periodic. The wall is assumed to be impermeable, such that the wall-normal mass flux of all species is identically zero. The jet nozzle shape is round with a diameter, $d=1 \mathrm{~mm}$. The center of the nozzle is located on the spanwise symmetry plane, $z=L_{z} / 2$, and at a streamwise location of $x=5.5 \mathrm{~mm}$. The governing equations are discretized using $1408 \times 1080 \times 1100$ Cartesian mesh points in the streamwise $x$, transverse $y$ and spanwise $z$ directions, respectively. The mesh is uniform in the $x$ and $z$ directions and non-uniform in the $y$ direction to satisfy the resolution requirements near the solid wall located at $y=0$. The non-uniform mesh 
is refined in the wall-normal direction near the wall using a hyperbolic tanh mapping, resulting in an increasing wall normal spatial resolution of 10.2 to $24.3 \mu \mathrm{m}$. The first point off the wall is at $y / \delta_{\text {vis }}=0.5$, where $\delta_{\text {vis }}$ is the thickness of the viscous sublayer of the boundary layer. There are two points within $y / \delta_{v i s}=1$ and 13 points within $y / \delta_{v i s}=10$, satisfying the resolution requirements in the viscous layer [26]. The spatial resolution of the present DNS yields $k_{\max } \eta=1.3$ where $k_{\max }$ is the maximum wave number in the domain and $\eta$ is the mean Kolmogorov length scale, ensuring that the smallest scales of turbulence are well resolved. A detailed analysis on numerical resolution for the present JICF configuration is reported in Ref. [15].

The jet fluid issues into a cross-flow and is comprised of $\mathrm{H}_{2}$ and $\mathrm{CO}$ species with the following volumetric ratios: (i) 1:0.06 (CO-lean) and (ii) 1:1 (COrich), while the cross-flow is air. The $\mathrm{H}_{2} / \mathrm{CO}$ composition for the CO-lean case corresponds to a typical syngas composition after WGS [27]. The jet and cross-flow mixtures are preheated to 420 and $750 \mathrm{~K}$, respectively. These temperatures are within the nominal temperature range of the fuel stream and of the combustion air delivered by the compression stage in large, stationary gas turbines for power generation in the 200-400 MW range, although atmospheric pressure conditions are considered in the present work due to the prohibitive computational cost of spatial resolution at higher pressure. The fuel mixtures issue into the cross-flow of air from the round hole flush mounted with the solid surface. The jet bulk velocities, $u_{j}$, are 250.0 and $176.0 \mathrm{~m} / \mathrm{s}$, respectively for the CO-lean and the CO-rich cases, maintaining a constant momentum flux ratio, $R_{m} \equiv\left[\left(\rho_{j} u_{j}^{2}\right) /\left(\rho_{c f} u_{c f}^{2}\right)\right]^{0.5}, 3.45$, where $\rho_{j}$ and $\rho_{c f}$ are the densities of jet and cross-flow mixtures. The jet Reynolds number, $R e_{j}=u_{j} d / \nu$, corresponding to these parameters is 3810 for the CO-lean and 4430 for the CO-rich cases, where $\nu$ is the kinematic viscosity of the jet fluid. In the DNS, a symmetric, top-hat-shaped profile is assigned to the jet wall-normal velocity component while the other two components are set to zero. The free-stream velocity of the cross-flow, $u_{c f}$, is $56.5 \mathrm{~m} / \mathrm{s}$ for both cases. As described in detail in a previous paper [15], the cross-flow velocity yields a Reynolds number $R e_{\delta_{99 \%}}$ in the range of $8200-8900$, where $R e_{\delta_{99 \%}}$ is defined based on $u_{c f}$, the boundary layer thickness $\delta_{99 \%}$ defined as the height at which the streamwise velocity reaches $99 \%$ of $u_{c f}$ and the kinematic viscosity of air.

New et al. (2006) [28] experimentally compared the behavior of top-hat and parabolic JICF arrangements at similar momentum ratios and found that while the time-averaged flows exhibit many similar features, the thinner 
shear layer in the top-hat profile led to earlier onset of more coherent leadingedge and lee-side vortices. From the perspective of attempting to generate a database for model development, an additional benefit of using a top-hat profile, such as the one that arises from nozzle flow, is that the jet boundary condition can be unambiguously specified and the results are not dependent on assumptions about the nature of - or interactions with - a notional pipe upstream of the jet inlet, such as would be the case for a parabolic profile. Turbulent velocity fluctuations are not imposed on the jet due to its relatively low Reynolds number. The latter boundary condition specification relies on the assumption that jet velocity fluctuations in themselves ultimately play a minor role in the transverse jets behavior compared to the approaching cross-flow turbulence, the turbulence generated by the interaction of the jet with the cross-flow, and the jet wall-normal velocity mean profile itself [28].

The cross-flow of air enters the domain from a non-reflecting inflow boundary at $x=0$. The velocity fluctuations imposed at the cross-flow inlet are obtained from temporal sampling of time-evolving turbulence at a fixed streamwise location in an auxiliary DNS of inert turbulent boundary layer flow with the same cross-flow velocity, $u_{c f}$, carried out separately. This approach, which allows eddies to "evolve" on the boundary, provides a more realistic description of the incoming turbulence compared with the usual practice of convecting an isotropic turbulence field at one time instant into the domain by using Taylors hypothesis. The simulation used to generate the inflow data is described in detail in [15].

The initial flow field is also obtained from the auxiliary DNS [15]. After the initialization of the combustion DNS domain, an intermediate mesh of $704 \times 540 \times 550$ is used to ignite the flame and flush the ignition transients out of the computational domain. Forced ignition is implemented by placing an ignition source across the fuel plume for a short duration of $8 \mu$ s before it is removed. The ignition source is obtained by smoothly imposing the adiabatic flame temperature and burnt composition in a cylindrical region of the domain characterized by a diameter of $0.25 \mathrm{~mm}$. The ignition source is placed at $x=6 \mathrm{~mm}$ and $y=1.5 \mathrm{~mm}$ and extends throughout the spanwise direction. Once all of the fluid present in the domain when the ignition source is removed exits through the outflow boundary, the solution is mapped onto the production mesh described above. Subsequently, the simulation is continued using a time step of $4 \mathrm{~ns}$ for 9 flow-through times, and $N_{t}=$ 247 (CO-lean) and 229 (CO-rich) instants of the field data are collected for the present analysis during the last flow-through period. The simulation is 
performed on 96000 cores of Cray XE6 (Hopper) at the National Energy Research Scientific Computing Center. The CPU cost is approximately 7.8 million CPU hours per flow-through time.

\section{Results}

\subsection{Flame stabilization characteristics}

Using advanced laser diagnostics a previous experimental study [29] revealed the existence of two flame branches in a JICF configuration: a persistent branch located upstream and referred to as a "lee-stabilized" branch and a second more intermittent branch located downstream on the windward side, referred to as a "ifted" branch. Based on the description in Ref. [4], both branches are comprised of stratified partially-premixed (triple) flames similar to those observed in conventional lifted co-flowing flames. On the basis of earlier qualitative observations reported in Ref. [14], we anticipate that the present $\mathrm{CO} / \mathrm{H}_{2}$ flame will anchor where the conditions are favorable in terms of fluid velocities and mixture preparation. As for mixture preparation, in the near field of the JICF the intense fine scale turbulence increases micro-mixing rates and provides a sufficiently homogeneous mixture resulting in flame stabilization via partially premixed combustion $[15,16]$. Accordingly, if turbulent mixing, which scales with the momentum flux ratio $R_{m}$, is kept constant, the laminar flame speed $S_{L}$ would have a dominant influence on flame stabilization $[30,29]$. Indeed, the fluid velocity component normal to the flame base is scaled by $S_{L}$ in JICF configurations [17]. Thus, as the amount of CO increases in the fuel stream, the laminar flame speed decreases, and one might expect the flame to anchor progressively farther downstream.

Figure 2 shows two sampling regions and the jet trajectory superimposed on the instantaneous and averaged heat release rate, $Q^{\circ}$ and $\bar{Q}^{\circ}$, and velocity magnitude, $\|\mathbf{u}\|^{*}$ and $\|\widetilde{\mathbf{u}}\|^{*}$ iso-contours in the spanwise mid plane. Here, the superscript "०" denotes normalization using the maximum value in a given 2D plane and "*" denotes normalization using a jet parameter(s) such as $d$ and/or $u_{j}$. Also, $\widetilde{q}$ refers to a Favre average of a quantity $q$ obtained using its Reynolds average $\bar{q}$ as $\widetilde{q}=\overline{\rho q} / \bar{\rho}$. The sampling regions, namely LW (leeward) and WW (windward), are located just downstream of the jet exit bounded by the jet trajectory having dimensions of $2 d$ (distance from the jet center line) $\times 0.2 l_{j}$, where $l_{j}$ is the length from the jet exit to the $x$ outflow boundary along the jet center line. The jet trajectory is constructed based 
on the mean velocity field. As described below, the LW sampling region is where most of the interesting flame stabilization phenomena exist. DNS results are presented at the symmetric spanwise mid-plane $\left(z \sim L_{z} / 2\right)$ since the most upstream reaction zones are located. The mean and instantaneous heat release rate fields in Fig. 2 show that there are two intense heat releasing regions similar to those observed in experiments [29]. These two regions correspond to the flame edges of the two branches. In the present configuration, however, the triple flame structure suggested by Ref. [4] is not apparent since these flame branches (lean and rich premixed and diffusion flames) interact and merge with each other. However, as described below each high heat release rate region spans lean, stoichiometric and rich mixtures. Additional mean fields (temperature, $Y_{\mathrm{CO}}, Y_{\mathrm{H}_{2}}$ and $Y_{\mathrm{OH}}$ for both CO-lean and CO-rich cases) on the spanwise mid plane are also provided as supplemental material.

The normalized velocity magnitude iso-contours in Figs. 2 show that the location and shape of the low-velocity region do not change significantly between the two cases. Thus, flame stabilization could be determined based on the laminar flame speeds $S_{L}, 2.92 \mathrm{~m} / \mathrm{s}$ for the CO-lean and $1.54 \mathrm{~m} / \mathrm{s}$ for the CO-rich mixtures under stoichiometric conditions with a reactant temperature of $420 \mathrm{~K}$ at atmospheric pressure. The ratio, $S_{L} / u_{j}$, is 0.0117 and 0.00875 for the CO-lean and CO-rich cases, respectively. However, both instantaneous and mean heat release rate fields in Fig. 2 show that the flame base exists at a similar location $\left(x^{*} \sim 7\right.$ and $\left.y^{*} \sim 2\right)$ for both cases. Also, the intense $\bar{Q}^{\circ}$ in the LW sampling region is located just outside of the $\|\widetilde{\mathbf{u}}\|^{*}=0.4$ iso-contour, indicating that both CO-lean and CO-rich flames are capable of stabilizing where $\|\widetilde{\mathbf{u}}\|^{*}<0.4$, corresponding to $100 \mathrm{~m} / \mathrm{s}$ for the CO-lean and $70.4 \mathrm{~m} / \mathrm{s}$ for the CO-rich cases. The ratio of flame base flow velocities for the two cases is substantially smaller than the ratio of $S_{L}$ noted above. These results suggest that scaling based on nominal flame speed is irrelevant to the flame stabilization location for syngas fuel considered in the present $\mathrm{CO} / \mathrm{H}_{2}$ range unlike that observed in previous measurements [17]. The flame stabilization mechanism involves additional complexity for multi-component fuel flames with varying molecular transport properties.

Based on the mean field in Fig. 2, the most upstream point of large heat release rate is located near/inside the small velocity region enclosed by $\|\widetilde{\mathbf{u}}\|^{*}=0.4$ iso-contours in the LW region. Thus, to further understand the flame stabilization mechanism in syngas combustion, the local mixture preparation in the LW region prior to combustion needs to be understood. For this purpose the Chemical Explosive Mode (CEM), $\lambda_{\exp }\left(\mathrm{s}^{-1}\right)$, is em- 
ployed [31]. The CEM is the largest positive eigenvalue of the reaction rate Jacobian, which is a reciprocal of the CEM time scale. The normalized CEM is defined here as:

$$
\Lambda_{\text {exp }}=\operatorname{sign}\left(\lambda_{\text {exp }}\right) \times \log _{10}\left[\max \left(1,\left|\lambda_{\text {exp }}\right|\right)\right] .
$$

Positive values of CEM indicate that the mixture is "explosive", although auto-ignition may not proceed in the presence of losses due to turbulent mixing and molecular diffusion. The CEM value increases as the mixture approaches the preheat zones, and peaks where intense chemical reactions commence. Once intense chemical reactions begin, the CEM decreases and attains negative values in the burnt mixture. Thus, local mixtures exhibiting maximum possible CEM are located in a region of very small CEM gradient, $\left\|\nabla \Lambda_{\text {exp }}\right\| \sim 0$ with $\Lambda_{\text {exp }} \gg 0$. Based on the CEM time scale, "Explosive Zones" (EZ) are identified using the following criteria:

$$
\Lambda_{\text {exp }} \geq 0.95 \Lambda_{\text {exp } \max },\left\|\nabla \Lambda_{\text {exp }}\right\| \leq 0.01\left\|\nabla \Lambda_{\text {exp }}\right\|_{\max },
$$

where the subscript "max" is a maximum value in a given 2D plane. Mixtures satisfying these conditions possess or nearly attain positive peak CEM locally and the local conditions are expected to be favorable for combustion. Note that mixtures classified as "explosive zones" will not necessarily involve explosive behavior. A laminar premixed flame front also exhibits "explosive" characteristics even though it is "propagative". An explosive zone identifies local mixture conditions that are well prepared and about to produce intense heat release rate. The probability of finding explosive zones is also defined as:

$$
p_{E Z}(\mathbf{x})=\frac{1}{N_{t}} \sum_{n=1}^{N_{t}} \delta_{E Z}(\mathbf{x}, t), \delta_{E Z}= \begin{cases}1, & \text { if }(\mathbf{x}, t) \in \mathrm{EZ} \\ 0, & \text { otherwise }\end{cases}
$$

where $N_{t}$ is the number of instantaneous DNS snapshots for each case. Figure 3 shows $p_{E Z}$ in the spanwise mid-plane for the CO-lean and the CO-rich cases. For both cases, there are two probable explosive zones (see the yellowredish colors in $p_{E Z}$ ) that are also observed in the $\bar{Q}^{\circ}$ field in Fig. 2. The upstream zone is located inside the LW sampling region and the second zone is located further downstream, spanning both the windward and leeward sides. The upstream explosive zones exist primarily on the lean side characterized by a lean partially premixed flame in a conventional lee-stabilized branch [29], while the intense heat release rate regions (see $\bar{Q}^{\circ}=0.8,0.7$ contour line in Fig. 3) in the flame base exist slightly on the rich side. Clearly, 
the upstream probable explosive zones, located slightly upstream of the high heat release rate, exist at a similar location between the two cases. The almost identical flame base locations between the two cases observed in Fig. 2 may be a consequence of similar locations of mixture preparation occurring prior to intense chemical reactions. Thus, it is of interest to understand the factors which contribute to the local mixture preparation in addition to turbulent mixing. These factors may illuminate the insensitivity of the flame base location to the fuel composition in syngas fuel composition.

\subsection{Differential diffusion and turbulent mixing}

Previous studies have reported the presence of increased micro-mixing caused by intense fine scale turbulence in the near field of a JICF $[15,16]$. Since such micro-mixing could help to create favorable homogeneous mixtures for flame stabilization, the turbulent scalar flux, $\widetilde{\left\|\mathbf{u}^{\prime \prime}\right\| \xi^{\prime \prime}}{ }^{*}$, is examined in Figs. 4a and 4b for the CO-lean and CO-rich cases, respectively. Here, $\xi$ is the mixture fraction based on Bilger's definition [32] and $\xi^{\prime \prime}$ is its Favre fluctuation. Consistent with previous studies, intense turbulent mixing regions are observed near the jet exit starting from $y^{*} \sim 0.5$ for both cases. The probable explosive zones $\left(p_{E Z}^{\circ} \geq 0.5\right)$ exist in regions of relatively small

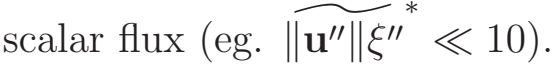

Similar to the effect of turbulence on mixing, scalar mixing can also be carried out through transport related to scalar dissipation rate of mixture fraction, $N_{\xi}=D_{\xi} \partial \xi / \partial x_{i} \partial \xi / \partial x_{i}$, and differential diffusion of species [13]. Here, $D_{\xi}$ is the mixture fraction diffusivity taken to be the thermal diffusivity in the present analysis. The mean scalar dissipation rate can be scaled using turbulent kinetic energy and its dissipation rate. Since these turbulence characteristics are not significantly different in the present DNS cases given the constant $R_{m}$, the mean scalar dissipation rate has qualitatively similar characteristics between the two flames as shown in Figs. 4c and 4d. As expected, one of the favorable mixture conditions for flame anchoring is having sufficiently low scalar dissipation rates since high dissipation rate delays the reactions via thermal and species concentration loss. The present flames also exhibit this preference by the explosive zones clearly, although the peak heat release rate region exists in a high scalar dissipation region since adequate mixing is required for reaction to occur as will be described in detail below.

As for differential diffusion, previous studies have shown that fuel mixtures comprised of hydrogen and hydrocarbons are prone to significant differ- 
ential diffusion effects in both non-premixed non-reacting [12] and reacting $[13,33]$ flows. Thus, differential species transport could also contribute to the formation of a favorable mixture pocket in the LW region. For the purposes of the present analysis, the extent of differential diffusion is quantified by $[12,13]$ :

$$
\xi_{\mathrm{HC}}=\xi_{\mathrm{H}}-\xi_{\mathrm{C}} .
$$

Here, $\xi_{\mathrm{H}}$ and $\xi_{\mathrm{C}}$ are the elemental mixture fractions of $H$ and $C$ defined as:

$$
\xi_{\mathrm{H}}=\frac{Z_{\mathrm{H}}-Z_{\mathrm{H}, o}}{Z_{\mathrm{H}, f}-Z_{\mathrm{H}, o}} ; \xi_{\mathrm{C}}=\frac{Z_{\mathrm{C}}-Z_{\mathrm{C}, o}}{Z_{\mathrm{C}, f}-Z_{\mathrm{C}, o}},
$$

where $Z_{\mathrm{H}}$ and $Z_{\mathrm{C}}$ are the elemental mass fractions of $\mathrm{H}$ and $\mathrm{C}$ and the subscripts, $f$ and $o$, denote the corresponding values in the fuel and oxidizer streams, respectively. Since this quantification of differential diffusion between $\mathrm{H}$ atom containing species and carbon containing species is based on elemental mass fractions, the interpretation of $\xi_{\mathrm{HC}}$ is straightforward, even in the presence of chemical reactions. A positive value of $\xi_{\mathrm{HC}}$ indicates an excess of hydrogen containing species compared to carbon containing species relative to that present in the fuel stream and vice-versa, purely due to differential molecular transport. Favre averaged $\xi_{\mathrm{HC}}$ is shown in Figs. $4 \mathrm{e}$ and $4 \mathrm{f}$ for the CO-lean and CO-rich cases. Clearly, the probable explosive zones inside LW exist in a relatively large $\xi_{\mathrm{HC}}$ region for both cases. Negative values of $\xi_{\mathrm{HC}}$ are observed in the shear zone since H-related species are diffused away due to differential diffusion in this region. The $R e_{\lambda}=80$ contour lines overlaid on the $\xi_{\mathrm{HC}}$ field in Figs. $4 \mathrm{e}$ and $4 \mathrm{f}$ show the relation between differential diffusion and local turbulence. Here, $R e_{\lambda}$ is the Reynolds number based on the Taylor microscale $\lambda$, and $\lambda$ is obtained as:

$$
\lambda=\frac{1}{3} \sum_{i=1}^{3} \lambda_{i}, \lambda_{i}=\sqrt{\widetilde{u_{i}^{\prime \prime 2}} / \widetilde{\left(\frac{\partial u_{i}^{\prime \prime}}{\partial x_{i}}\right)^{2}}}
$$

where $u_{i}^{\prime \prime}$ is the fluctuation of $u_{i}$ from its Favre average. Due to the nature of the formulation for $R e_{\lambda}$, relatively large $R e_{\lambda}(>80)$ could appear in the shear layer where the turbulence is not fully developed. The $R e_{\lambda}>80$ regions are also observed outside the shear zones, although such samples occupy a small volume for the CO-lean case compared to the CO-rich case. This difference is due to the constant $R_{m}$ conditions which results in different $R e_{j}$ between the two cases. However, in spite of the large $R e_{\lambda}$ regions, the locations of the 
explosive zones in the CO-rich case remains similar to that in the CO-lean case. Thus, local turbulence alone does not seem to have a significant impact on the explosive zone locations compared to differential diffusion effects. The spatial relationship between explosive zones and positive differential diffusion is also revealed in several $x-z$ planes at different $y$ locations as shown in Fig. 5 . As clearly seen, the most upstream explosive zones (see Fig. 5a) on average tend to appear near the spanwise mid-plane. The upstream explosive zones prefer high $\tilde{\xi}_{\mathrm{HC}}$ as discussed above, but not all high $\tilde{\xi}_{\mathrm{HC}}$ regions are associated with explosive zones. For example, for both sides of the jet in the leeward side (see arrows in Fig. 5a) high values of $\tilde{\xi}_{H C}$ are also observed, due to the high curvatures of the scalar iso-surfaces. However, explosive zones do not exist at these locations since the mean velocity is not small enough to achieve sufficient residence time for mixture preparation. At downstream positions (Figs. 5b-5e), the shape of $\xi$ iso-lines approaches a "kidney" shape as the CVP develops. This shape produces a high concentration of $\mathrm{H}$ due to the mean curvature and scalar gradient at the spanwise mid-plane position (see arrow in Fig. 5e), although this effect is insignificant for near field flame stabilization. The explosive regions downstream also show the evolution of the CVP. The effect of strain rate tangent to $\xi$ iso-surfaces on mixture preparation through differential diffusion [33] (not shown) is also found to be insignificant since intense tangential strain exists primarily on the windward side of the jet.

To examine the preference of explosive zones and differential diffusion relative to local turbulence, conditional averages conditioned on the Reynolds number, $\left\langle\xi_{\mathrm{HC}} \mid R e_{\lambda}\right\rangle$ and $\left\langle\left\|\mathbf{u}^{\prime \prime}\right\| \xi^{\prime \prime *} \mid R e_{\lambda}\right\rangle$, collected from the LW sampling region are presented in Figs. $6 \mathrm{a}$ and $6 \mathrm{~b}$. For $R e_{\lambda} \leq 10$, the value of $\xi_{\mathrm{HC}}$ is very small $\left(\xi_{\mathrm{HC}} \sim 0.01\right.$ or less) since these samples are located inside the jet where the elemental mixture fractions of both $\mathrm{H}$ and $\mathrm{C}$ are almost unity. Outside the jet, the amount of excess $H$ decreases while $\left\|\mathbf{u}^{\prime \prime}\right\| \xi^{\prime \prime *}$ increases with an increase of $R e_{\lambda}$ for $R e_{\lambda} \leq 80$ for both CO-lean and CO-rich cases. However, the trends of differential diffusion relative to $R e_{\lambda}$ are different between the two cases for $R e_{\lambda}>80$. For the CO-lean case (Fig. 6a), the value of $\xi_{\text {HC }}$ continually decreases with $R e_{\lambda}$ while $\left\|\mathbf{u}^{\prime \prime}\right\| \xi^{\prime \prime *}$ remains important. Such behavior is consistent with the asymptotic relation, $\xi_{H C} \propto 1 / R e_{t}$ at high Reynolds number [34]. Thus, turbulent mixing is predominant and the effect of differential diffusion becomes subordinate in this region. In contrast, the behavior of $\xi_{\mathrm{HC}}$ at $R e_{\lambda}>80$ is opposite for the CO-rich case; $\xi_{\mathrm{HC}}$ increases with an increase of $R e_{\lambda}$ while $\left\|\mathbf{u}^{\prime \prime}\right\| \xi^{\prime \prime *}$ diminishes. Here, one should be careful about 
the interpretation of $\xi_{\mathrm{HC}}$ for $R e_{\lambda}>80$ in this figure. Most of these samples are located in the high $R e_{\lambda}$ regions outside the shear layer (see Fig. 4f) with sparse samples collected from inside the shear layer where turbulent mixing is more predominant than differential diffusion. In the high $R e_{\lambda}$ regions the scalar flux is low despite the relatively intense local turbulence. This is represented by the decreasing value of $\left\|\mathbf{u}^{\prime \prime}\right\| \xi^{\prime \prime *}$ at larger $R e_{\lambda}$ in Fig. 6b. As a result, differential diffusion becomes predominant compared to turbulent mixing in these regions. Thus, on average turbulent mixing and not Reynolds number is negatively correlated with differential diffusion over a range of $R e_{\lambda}$. This is also implied by a previous investigation into the relation between differential diffusion and global Reynolds number [13]. Also, when Karlovitz number $K a=\left(u_{\eta} \delta_{F}\right) /\left(\eta S_{L}\right)$ is used for conditioning (Figs. 6c and 6d), the increasing $\xi_{H C}$ with local turbulence level in the CO-rich case becomes less apparent and for large $K a, K a$ has a stronger negative correlation with $\xi_{H C}$. Here, $u_{\eta}$ is the Kolmogorov velocity scale and $\delta_{F}$ is the Zeldovich flame thickness.

Figure 6 also shows the volume averages of $\xi_{\mathrm{HC}}$ and $\left\|\mathbf{u}^{\prime \prime}\right\| \xi^{\prime \prime *}$ collected from the probable explosive zones $\left(p_{E Z}^{\circ} \geq 0.5\right)$ in the entire domain and from those in the LW sampling region to highlight the preferential location of the flame base with respect to the location of excess H. Generally, the explosive zones exist where $\xi_{\mathrm{HC}}$ and $\left\|\mathbf{u}^{\prime \prime}\right\| \xi^{\prime \prime *}$ have comparable values (see red and black circles), suggesting that the formation of reaction zones is favorable in locations with both sufficient amounts of turbulent mixing and excess $\mathrm{H}$ resulting from differential diffusion. Among all the explosive zones throughout the domain, those forming the flame base (EZ in LW) particularly have a strong preference to excess $\mathrm{H}$ over turbulent mixing for both cases (see red and black plus symbols). Thus, it is reasonable to conjecture that the small amount of $\mathrm{H}_{2}$ in the syngas fuel produces excess $\mathrm{H}$ in the $\mathrm{LW}$ region due to differential diffusion, which enhances the reactivity of the mixture that forms the flame base.

The relation between differential diffusion and turbulent mixing is more clearly seen in the scatter plot in $\widetilde{\xi_{\mathrm{HC}^{-}}}\left\|\widetilde{\mathbf{u}^{\prime \prime}}\right\| \xi^{\prime \prime} * \mid$ space shown in Figs. 7a (COlean) and $7 \mathrm{~b}$ (CO-rich). As described above, most of the explosive zones in the LW region (they are likely to form the flame base) are located where differential diffusion is predominant compared to turbulent mixing for both cases. Other non-EZ samples in the LW region (blue scatter) are distributed in a broad area with three branches which can be categorized as: relatively large excess $\mathrm{H}$ and small turbulent mixing (zone $[\mathrm{I}]$ ); reasonable or small 
excess $\mathrm{H}$ and relatively large turbulent mixing (zones [II]); and negligible $\widetilde{\xi_{\mathrm{HC}}}$ or excess $\mathrm{C}$ and large turbulent mixing (zone [III]). This classification is performed using the following criteria.

$$
\begin{aligned}
& \text { Zone }[\mathrm{I}]: \widetilde{\xi}_{H C} \geq 0.25 \widetilde{\xi}_{H C, \max } \text { and } \widetilde{\left\|\mathbf{u}^{\prime \prime}\right\| \xi^{\prime \prime}}{ }^{*} \leq 0.25\left\|\widetilde{\mathbf{u}}^{\prime \prime}\right\| \xi^{\prime \prime}{ }_{\max } \text {. } \\
& \text { Zone [II] : } \widetilde{\xi}_{H C} \geq 0.05 \widetilde{\xi}_{H C, \max } \text { and } \widetilde{\left\|\mathbf{u}^{\prime \prime}\right\| \xi^{\prime \prime}}{ }^{*}>0.25 \|{\widetilde{\mathbf{u}^{\prime \prime}}}{ }^{\prime \prime}{ }^{*}{ }_{\max } \text {. } \\
& \text { Zone [III] : } \widetilde{\xi}_{H C}<0.05 \widetilde{\xi}_{H C, \max } \text { and } \widehat{\left\|\mathbf{u}^{\prime \prime}\right\| \xi^{\prime \prime}}{ }^{*} \geq 0.25\left\|\widehat{\mathbf{u}}^{\prime \prime}\right\| \xi^{\prime \prime}{ }_{\max }^{*} \text {. }
\end{aligned}
$$

These criteria are arbitrary and are based on visual inspection of trends from Figs. 7a and 7b. However, the zones are insensitive to changes in the threshold values. For example, changes in values by $\pm 50 \%$ do not alter the conclusions addressed here. The locations of the zones [I]-[III] along with the probable explosive zones are shown with heat release rate contours in physical space in Figs. 7c and 7d. Clearly, the region of predominant differential diffusion (zone $[\mathrm{I}]$ ) is not collocated with the region of predominant predominant turbulent mixing (zone [III]). While the probable explosive zones are located in zone $[\mathrm{I}]$, the peak heat release in the LW region appears slightly downstream of zone [II] where both turbulent mixing and the level of excess $\mathrm{H}$ are substantial. These zone maps clearly suggest that differential diffusion plays a significant role in mixture preparation and initiating chemical reactions. This occurs prior to intense chemical reactions maintained by sufficient mixing due to turbulent motion and scalar dissipation rate, and by a small amount of radicals and heat provided by the initiation in the explosive zones.

\subsection{Effects of differential diffusion on mixture preparation}

In the previous section it was shown that the explosive zones in the LW region, which is likely to be a flame base, prefer excess $\mathrm{H}$ to turbulent mixing. The present section addresses how differential diffusion contributes to mixture preparation prior to intense chemical reactions in JICF using the Takeno Flame Index (FI) in the near field. The Flame Index is a metric for the alignment between the gradient of fuel $\left(\mathrm{H}_{2}\right.$ and/or $\left.\mathrm{CO}\right)$ and oxidizer $\left(\mathrm{O}_{2}\right)$ mass fractions used to describe the mixing mode between the fuel and oxidizer [35]:

$$
\mathrm{FI}=\frac{\nabla\left(Y_{\mathrm{H}_{2}}+Y_{\mathrm{CO}}\right) \cdot \nabla Y_{\mathrm{O}_{2}}}{\left|\nabla\left(Y_{\mathrm{H}_{2}}+Y_{\mathrm{CO}}\right)\right|\left|\nabla Y_{\mathrm{O}_{2}}\right|} .
$$


In perfectly premixed and non-premixed mixtures, FI yields 1 and -1, respectively. Positive FI indicates that fuel and oxidizer are premixed, and otherwise, non-premixed. Figure 8 shows the mean Flame Index in the near field with mean velocity and $p_{E Z}$ iso-lines. The mixing mode shown in Fig. 8 seems to be consistent with the idea that flame stabilization is a result of micro-mixing that provides a sufficiently homogeneous mixture, but only for the CO-lean case. Such a positive FI exists where the velocity magnitude is sufficiently low and turbulent mixing is sufficiently high (but not too high; see Fig. 4a). In such a region there is sufficient residence time for mixing [14]. For the CO-rich case, however, the mean FI is negative for almost the entire LW region including the explosive zones, suggesting that mixing between fuel and oxidizer does not, on average, provide an "ideal condition" for the explosive zones. Note that the instantaneous fields (not shown) reveal the presence of positive FI locally in the LW region, similar to findings from an earlier JICF DNS study [14]. However, positive FI is not predominant in the CO-rich case resulting in an overall negative $\overline{\mathrm{FI}}$. To further investigate such inconsistent behaviors of FI between CO-lean and CO-rich cases, the Flame Index is obtained based on individual fuel species as:

$$
\mathrm{FI}_{\mathrm{H}_{2}}=\frac{\nabla Y_{\mathrm{H}_{2}} \cdot \nabla Y_{\mathrm{O}_{2}}}{\left|\nabla Y_{\mathrm{H}_{2}}\right|\left|\nabla Y_{\mathrm{O}_{2}}\right|}, \quad \mathrm{FI}_{\mathrm{CO}}=\frac{\nabla Y_{\mathrm{CO}} \cdot \nabla Y_{\mathrm{O}_{2}}}{\left|\nabla Y_{\mathrm{CO}}\right|\left|\nabla Y_{\mathrm{O}_{2}}\right|}
$$

The Flame Indices based on individual fuel species are shown in Fig. 9 with heat release rate and $p_{E Z}$ iso-lines. For the CO-lean case as shown in Fig. 9a, the more diffusive $\mathrm{H}_{2}$ species is mixed with the oxidizer before $\mathrm{CO}$ is, creating an ideal homogeneous mixture for the explosive zones ( $\operatorname{see} p_{E Z}^{\circ}=0.5$ envelope). In contrast, positive values of $\mathrm{FI}_{\mathrm{CO}}$ exists only near the intense heat release rate region where scalar and velocity gradients are high enough to enhance scalar mixing.

For the CO-rich case, the trend is more or less similar. The location of positive values of $\mathrm{FI}_{\mathrm{H}_{2}}$ exists in the vicinity of the $p_{E Z}^{\circ}=0.5$ envelope, while the value is generally negative for $\mathrm{FI}_{\mathrm{CO}}$, although its magnitude decreases near high scalar and velocity gradient zones. Without differential diffusion, $\mathrm{H}_{2}$ would behave similar to $\mathrm{CO}$ in Fig. 9b and the probable explosive zones would consequently locate further downstream, possibly resulting in dynamic flame blowout. This conclusion also holds when instantaneous FI, $\mathrm{FI}_{\mathrm{H}_{2}}$ and $\mathrm{FI}_{\mathrm{CO}}$ (not shown) are examined. Several quantities in the explosive zones in the LW region, which could potentially influence flame stabilization, are qualitatively compared with those in the WW and downstream regions in Ta- 
ble. 1. The ensemble averages clearly validate the importance of differential diffusion of mobile species such as $\mathrm{H}_{2}$ for premixing prior to combustion. The averaged value of $\mathrm{FI}_{\mathrm{H}_{2}}$ in the explosive zones shows the distinctive features in the LW region compared with the WW and downstream regions for both CO-lean and CO-rich cases while other quantities are not as distinctive as $\mathrm{FI}_{\mathrm{H}_{2}}$. While the present DNS are performed at moderate turbulent Reynolds numbers, even at much higher turbulent Reynolds numbers the fluid dynamical mechanism of anchoring of the JICF in the localized low velocity regions on the lee side of the jet are likely persist. Hence, differential molecular diffusion of hydrogen-enriched fuel blends in the flame anchoring region will remain important.

\section{Conclusions}

Three-dimensional DNS of a transverse syngas fuel jet in a turbulent boundary layer cross-flow of air shows the influence of increasing the concentration of $\mathrm{CO}$ relative to $\mathrm{H}_{2}$ on near field flame stabilization. The mean flame anchors at a similar location for the two cases counter to the trend suggested by their laminar flame speeds which is higher for the CO-lean condition. To identify local mixtures having preferable mixture conditions for flame anchoring, explosive zones are defined using the CEM timescale. The explosive zones related to flame stabilization are located in relatively low velocity regions, slightly upstream of the peak heat release rate region. The explosive zones are characterized by excess $\mathrm{H}$ transported by differential diffusion with relatively low turbulent mixing intensity. The conditional averages show that differential diffusion is negatively correlated with turbulent mixing, while Reynolds number alone is insufficient to estimate the positive differential diffusion effect. This complementary relationship is further explained through examination of a scatter plot of differential diffusion and turbulent mixing. Based on the clustering in the scatter plot, three zones are defined: [I] large excess $\mathrm{H}$ with small turbulent mixing; [II] reasonable or small excess $\mathrm{H}$ and large turbulent mixing; and [III] negligible $\widetilde{\xi_{\mathrm{HC}}}$ or excess $\mathrm{C}$ and large turbulent mixing. Clearly, the most probable explosive zones exist in zone [I]. Comparing these three zones with the heat release rate and probable explosive zones, it is found that differential diffusion predominantly plays an important role in mixture preparation and initiates chemical reactions, followed downstream by intense chemical reactions sustained by sufficient mixing owning to the turbulent motion and scalar dissipation rate 
in the zone [II]. Finally, the mechanism by which differential diffusion contributes to mixture preparation is investigated using the Flame Index. The mean Flame Index shows that the extent of premixing is not intense based on combined fuel species. However, the Flame Index based separately on $\mathrm{H}_{2}$ and $\mathrm{CO}$ species reveals that $\mathrm{H}_{2}$ has a significant contribution to premixing, particularly in the explosive zones in the upstream leeward region which are likely to be the flame base. Therefore, a small amount of $\mathrm{H}_{2}$ is able to diffuse much faster than $\mathrm{CO}$, creating a relatively homogeneous mixture which, together with its reactivity, helps to stabilize a flame at a similar location

regardless of the amount of $\mathrm{CO}$ present in the fuel for the present range of DNS conditions. Therefore, the near-field flame stabilization mechanism in a multi-component fuel JICF is not solely due to a balance between the flame speed and local fluid velocity, but differential diffusion of lighter species also plays an important role. This suggests that combustion simulation of a gas turbine should employ both turbulent and molecular mixing especially in the near field.

\section{Acknowledgments}

This research used computational resources of the Oak Ridge Leadership Computing Facility (OLCF) at Oak Ridge National Laboratory, and National Energy Research Scientific Computing Center (NERSC). OLCF is supported by the office of Science of the US Department of Energy under contract DEAC05-00OR22725. NERSC is supported by the Office of Science of the U.S. Department of Energy under contract DE-AC02-05CH11231. Sandia is a multiprogram laboratory operated by Sandia Corporation, a Lockheed Martin Company, for the United States Department of Energy under contract DE-AC04-94AL85000. The work at Sandia National Laboratories was supported by the Division of Chemical Sciences, Geosciences and Biosciences, Office of Basic Energy Sciences of the US Department of Energy. The work at SINTEF has been produced with support from the BIGCCS Centre, performed under the Norwegian research program Centres for Environmentfriendly Energy Research (FME). The authors acknowledge the following partners for their contributions: Gassco, Shell, Statoil, TOTAL, GDF SUEZ and the Research Council of Norway (193816/S60). 


\section{References}

[1] V. AA., Energy technology perspectives 2014 - harnessing electricity's potential, Tech. Rep. ISBN 978-92-64-20800-1, IEA (2014).

[2] T. Lieuwen, V. McDonell, D. Santavicca, T. Sattelmayer, Burner development and operability issues associated with steady flowing syngas fired combustors, Combustion Science and Technology 180 (2008) 11671190 .

[3] J. Fritz, M. Kröner, T. Sattelmayer, Flashback in a swirl burner with cylindrical premixing zone, ASME Journal of Engineering for Gas Turbines and Power 126 (2004) 276-283.

[4] D. J. Micka, J. F. Driscoll, Stratified jet flames in a heated (1390K) air cross-flow with autoignition, Combust. Flame 159 (3) (2012) 1205-1214.

[5] C. Mayer, J. Sangl, T. Sattelmayer, T. Lachaux, S. Bernero, Study on Operational Window of a Swirl Stabilized Syngas Burner under Atmospheric and High Pressure Conditions, in: Proceedings of ASME Turbo Expo 2011, June 6-10, 2011, Vancouver, Canada, American Society of Mechanical Engineers, 2011, pp. GT2011-45125.

[6] C. Eichler, T. Sattelmayer, Premixed flame flashback in wall boundary layers studied by long-distance micro-piv, Experiments in Fluids 52 (2) (2012) 347-360.

[7] A. Gruber, J. H. Chen, D. Valiev, C. K. Law, Direct numerical simulation of premixed flame boundary layer flashback in turbulent channel flow, J. Fluid Mech. 709 (2012) 516-542.

[8] A. Gruber, A. R. Kerstein, D. Valiev, C. K. Law, H. Kolla, J. H. Chen, Modeling of mean flame shape during premixed flame flashback in turbulent boundary layers, Proc. Combust. Inst. 35 (2) (2015) 1485-1492.

[9] T. F. Fric, A. Roshko, Vortical structure in the wake of a transverse jet, J. Fluid Mech. 279 (1994) 1-47.

[10] L. K. Su, M. G. Mungal, Simultaneous measurements of scalar and velocity field evolution in turbulent cross flowing jets, J. Fluid Mech. 513 (2004) 1-45. 
[11] S. Mupiddi, K. Mahesh, Direct numerical simulation of passive scalar transport in transverse jets, J. Fluid Mech. 598 (2008) 335-360.

[12] L. L. Smith, R. W. Dibble, L. Talbot, R. S. Barlow, C. D. Carter, Laser Raman scattering measurements of differential molecular diffusion in nonreacting turbulent jets of $\mathrm{H} 2 / \mathrm{CO} 2$ mixing with air, Phys. Fluids 7 (6) (1995) 1455.

[13] L. L. Smith, R. W. Dibble, L. Talbot, R. S. Barlow, C. D. Carter, Laser Raman scattering measurements of differential molecular diffusion in turbulent nonpremixed jet flames of $\mathrm{H}_{2} / \mathrm{CO}_{2}$ fuel, Combust. Flame 100 (1-2) (1995) 153-160.

[14] R. Grout, A. Gruber, C. Yoo, J. Chen, Direct numerical simulation of flame stabilization downstream of a transverse fuel jet in cross-flow, Proc. Combust. Inst. 33 (2011) 1629-1637.

[15] R. W. Grout, A. Gruber, H. Kolla, P.-T. Bremer, J. C. Bennett, A. Gyulassy, J. H. Chen, A direct numerical simulation study of turbulence and flame structure in transverse jets analysed in jet-trajectory based coordinates, J. Fluid Mech. 706 (2012) 351-383.

[16] H. Kolla, R. W. Grout, A. Gruber, J. H. Chen, Mechanisms of flame stabilization and blowout in a reacting turbulent hydrogen jet in crossflow, Combust. Flame 159 (8) (2012) 2755-2766.

[17] E. F. Hasselbrink, M. G. Mungal, Observations on the stabilization region of lifted non-premixed methane transverse jet flames, Proc. Combust. Inst. 27 (1998) 1167-1173.

[18] D. Han, M. G. Mungal, Stabilization in turbulent lifted deflected-jet flames, Proc. Combust. Inst. 29 (2002) 1889-1895.

[19] J. H. Chen, A. Choudhary, B. de Supinski, M. DeVries, E. R. Hawkes, S. Klasky, W. K. Liao, K. L. Ma, J. Mellor-Crummey, N. Podhorszki, R. Sankaran, S. Shende, C. S. Yoo, Terascale direct numerical simulations of turbulent combustion using S3D, Comput. Sci. Discov. 2 (2009) $1-31$.

[20] E. R. Hawkes, R. Sankaran, J. C. Sutherland, J. H. Chen, Scalar mixing in direct numerical simulations of temporally evolving plane jet flames 
with skeletal $\mathrm{CO} / \mathrm{H}_{2}$ kinetics, Proc. Combust. Inst. 31 (1) (2007) 16331640 .

[21] R. Kee, F. Rupley, E. Meeks, J. Miller, Chemkin III: A Fortran chemical kinetics package for the analysis of gas-phase chemical and plasma kinetics, Sandia National Laboratories SAND96-8216.

[22] R. J. Kee, G. Dixon-Lewis, J. Warnatz, M. E. Coltrin, J. A. Miller, A Fortran computer code package for the evaluation of gas-phase multicomponent transport properties, Sandia National Laboratories SAND86-8246.

[23] C. A. Kennedy, M. H. Carpenter, Several new numerical methods for compressible shear-layer simulations, Appl. Numer. Math. 14 (4) (1994) 397-433.

[24] T. Poinsot, S. Lele, Boundary conditions for direct simulations of compressible viscous flows, J. Comput. Phys. 101 (1992) 104-129.

[25] J. C. Sutherland, C. A. Kennedy, Improved boundary conditions for viscous, reacting, compressible flows, J. Comput. Phys. 191 (2003) 502524 .

[26] R. D. Moser, J. Kim, N. N. Mansour, Direst numerical simulation of turbulent channel flow up to $\operatorname{Re}_{\tau}=590$, Phys. Fluids 11 (4) (1999) 943.

[27] N. S. Siefert, S. Litster, Exergy and economic analysis of advanced IGCC-CCS and IGFC-CCS power plants, Applied Energy 107 (2013) $315-328$.

[28] T. H. New, T. T. Lim, S. C. Luo, Effects of jet velocity profiles on a round jet in cross-flow, Experiments in Fluids 40 (2006) 859-875.

[29] A. Steinberg, R. Sadanandan, C. Dem, P. Kutne, W. Meier, Structure and stabilization of hydrogen jet flames in cross-flows, Proc. Combust. Inst. 34 (1) (2013) 1499-1507.

[30] R. Huang, J. Chang, The stability and visualized flame and flow structures of a combusting jet in cross flow, Combust. Flame 98 (3) (1994) $267-278$. 
[31] T. F. Lu, C. S. Yoo, J. H. Chen, C. K. Law, Three-dimensional direct numerical simulation of a turbulent lifted hydrogen jet flame in heated coflow: a chemical explosive mode analysis, J. Fluid Mech. 652 (2010) 45-64.

[32] R. W. Bilger, S. H. Stårner, R. J. Kee, On reduced mechanism for methane-air combustion in nonpremixed flames, Combust. Flame 80 (1990) 135-149.

[33] J. C. Sutherland, P. J. Smith, J. H. Chen, Quantification of differential diffusion in nonpremixed systems, Combust. Theory Model. 9 (2) (2005) 365-383.

[34] R. W. Bilger, Molecular transport effects in turbulent diffusion flames at moderate reynolds number, AIAA J. 20 (7) (1982) 962-970.

[35] H. Yamashita, M. Shimada, T. Takeno, A numerical study on flame stability at the transition point of jet diffusion flames, Symp. (Int.) Combust. 26 (1) (1996) 27-34. 


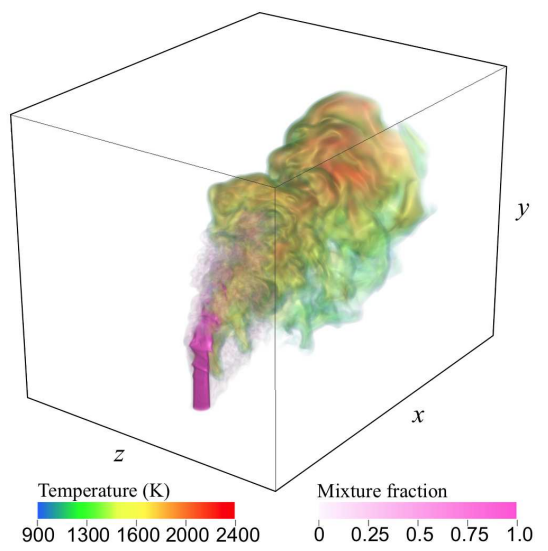

Figure 1: Volume rendering of instantaneous temperature and mixture fraction fields for the CO-lean case. 


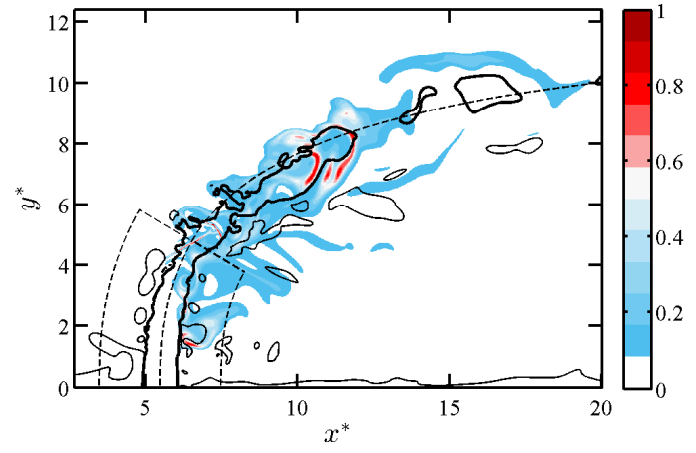

(a)

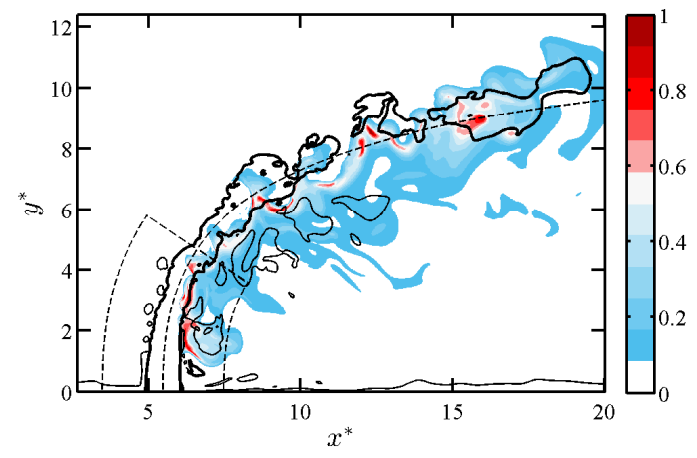

(c)

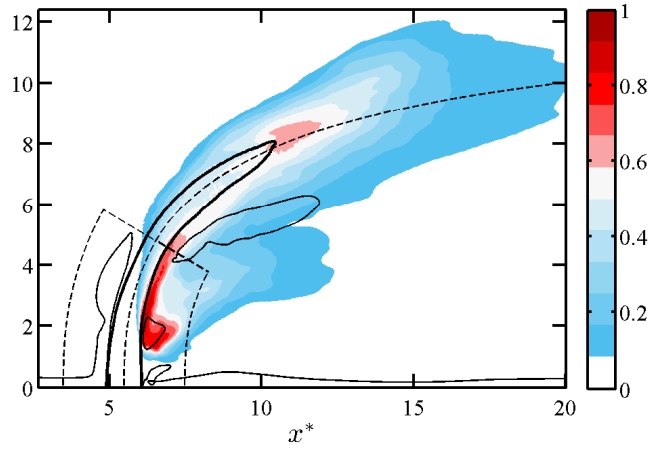

(b)

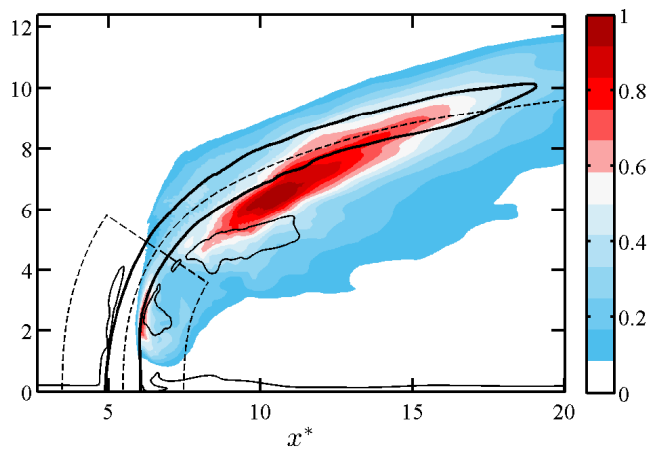

(d)

Figure 2: (a, c) Instantaneous $Q^{\circ}$ and (b, d) mean $\bar{Q}^{\circ}$ heat release rate in the mid-plane for CO-lean (a, b) and CO-rich (c, d) cases. Solid lines: iso-lines of $\|\mathbf{u}\|^{*}=0.1$ (thin) and 0.4 (bold) (a, c) and $\|\widetilde{\mathbf{u}}\|^{*}=0.1$ (thin) and 0.4 (bold) (b, d). Thin dashed lines show the leeward (LW) and windward (WW) sampling domains and mean jet trajectory. 


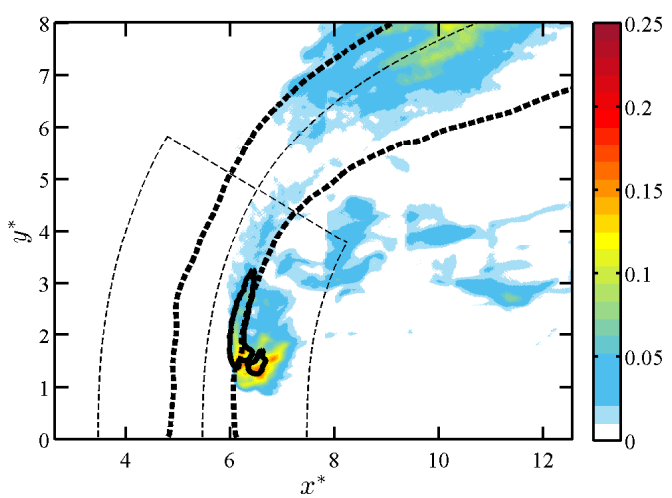

(a)

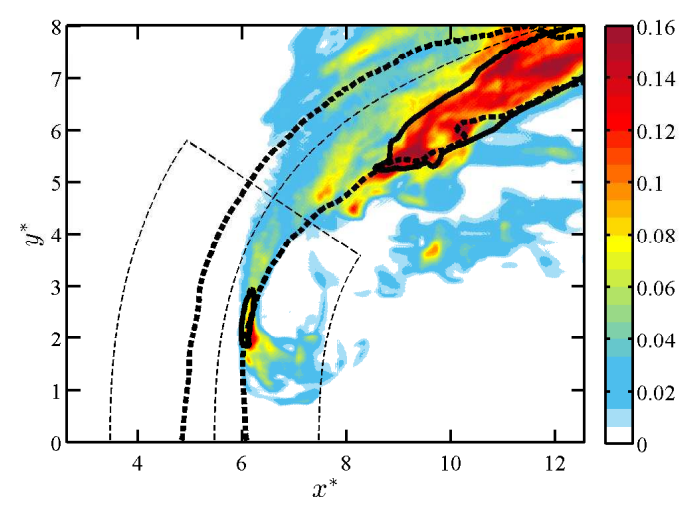

(b)

Figure 3: Iso-lines of stoichiometric mixture fraction $\xi_{s t}(\xi=0.188)$ for the CO-lean (a) and $(\xi=0.293)$ for the CO-rich (b) cases (bold dashed line) and the mean heat release rate $\bar{Q}^{\circ}=0.8$ for CO-lean (a) and 0.7 for CO-rich (b) cases (bold solid line) overlaid on the probability of finding the explosive zones $p_{E Z}$. 


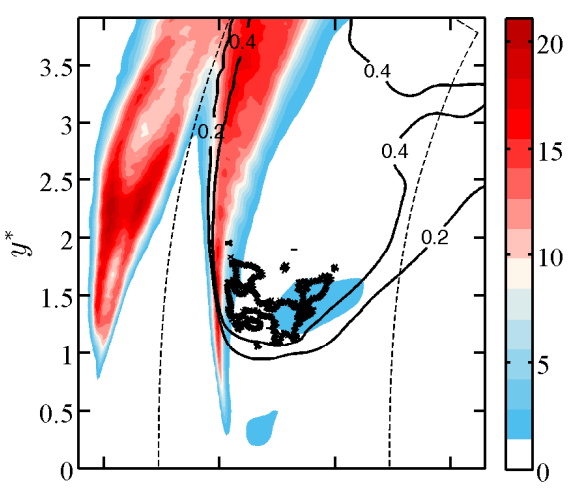

(a)

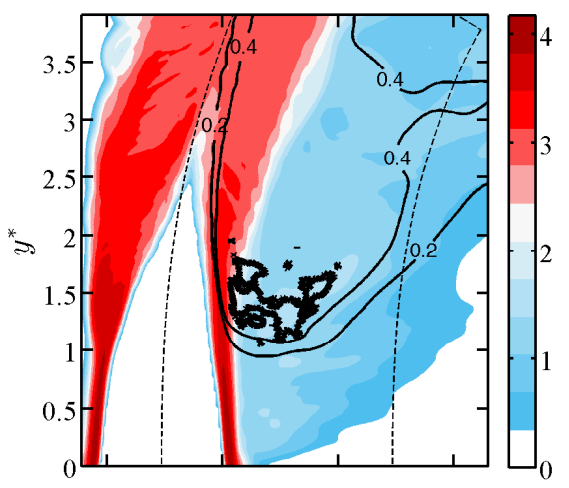

(c)

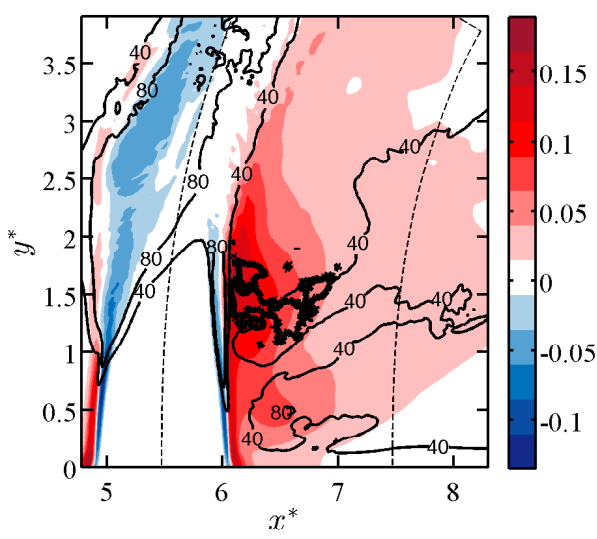

(e)

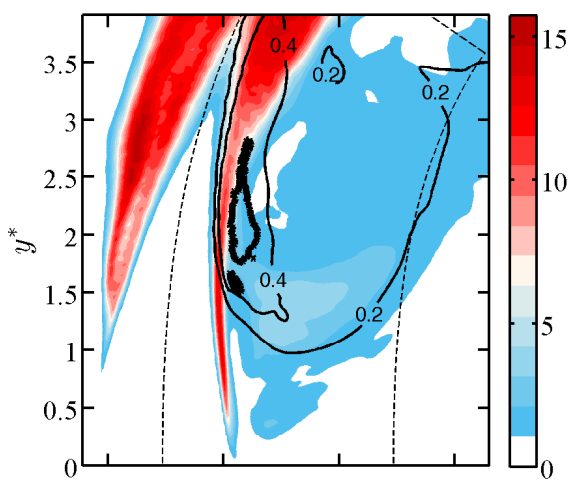

(b)

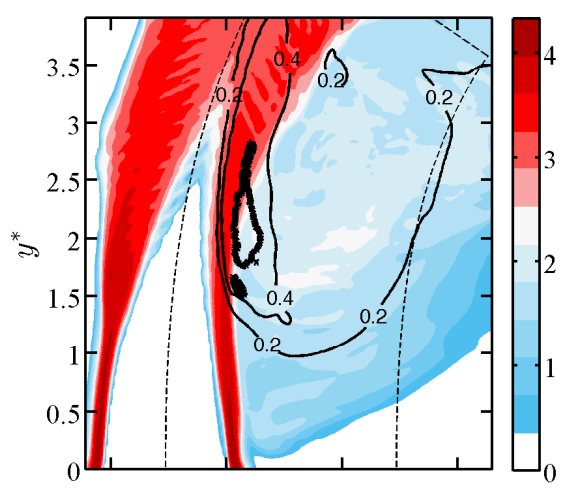

(d)

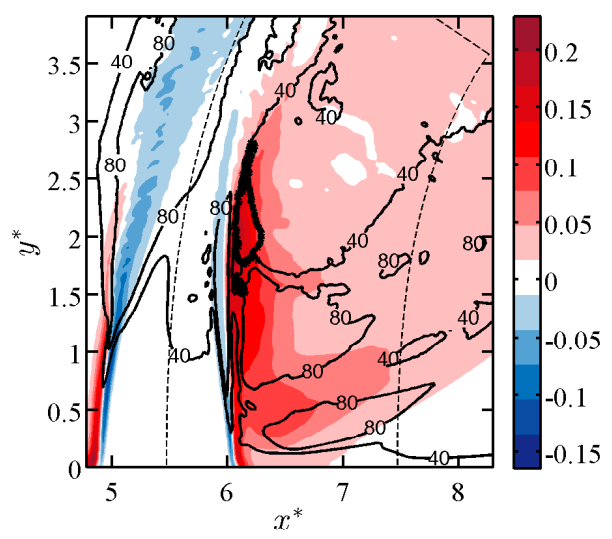

(f)

Figure 4: Color contours of (a, b) the mean turbulent scalar flux $\|{\widetilde{\mathbf{u}^{\prime \prime}} \| \xi^{\prime \prime}}^{*}$, (c, d) the logarithm of the scalar dissipation rate, $\log _{10}\left(\widetilde{N}_{\xi}(1 / s)\right)$ and (e, f) $\widetilde{\xi}_{\mathrm{HC}}$ for the CO-lean (a, $\mathrm{c}, \mathrm{e}$ ) and the CO-rich (b, d, f) cases. Iso-lines of mean heat release rate $\bar{Q}^{\circ}=0.2,0.4$ (thin solid line, (a, b, c, d)), Taylor Reynolds number $R e_{\lambda}=40,80$ (thin solid line, (e, f)) and the probability of finding explosive zones $p_{E Z}^{\circ}=0.5$ (bold solid line) are also overlaid. The thin dashed lines show the leeward (LW) and windward (WW) sampling domains and the mean jet trajectory. 


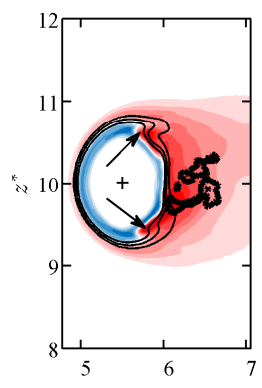

(a)

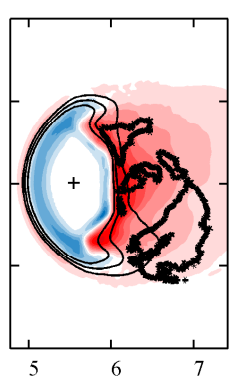

(b)

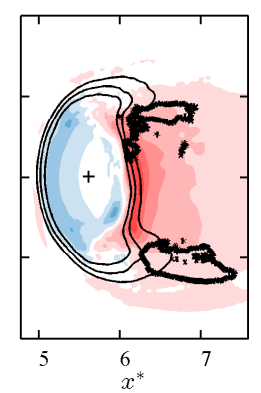

(c)

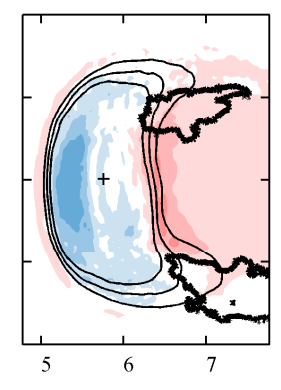

(d)

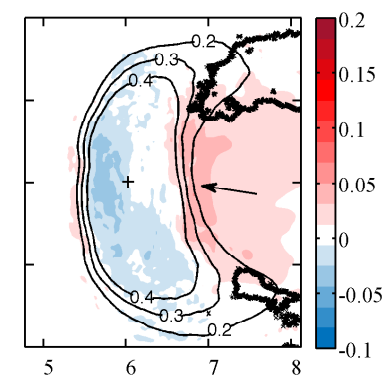

(e)

Figure 5: Mean mixture fraction iso-lines $(\tilde{\xi}=0.2,0.3,0.4)$ overlaid on the differential diffusion elemental mixture fraction $\tilde{\xi}_{H C}$ in the $x-z$ slices at different $y$ positions, (a) $y^{*}=1.20$, (b) $y^{*}=1.69$, (c) $y^{*}=2.20$, (d) $y^{*}=2.88$, (e) $y^{*}=3.75$, for the CO-lean case. Bold line shows explosive zones. The mean jet trajectory is also denoted by the + symbol. 


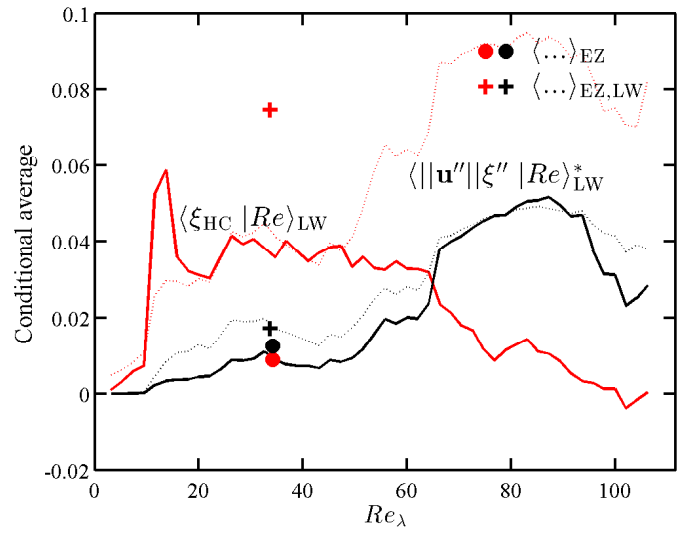

(a)

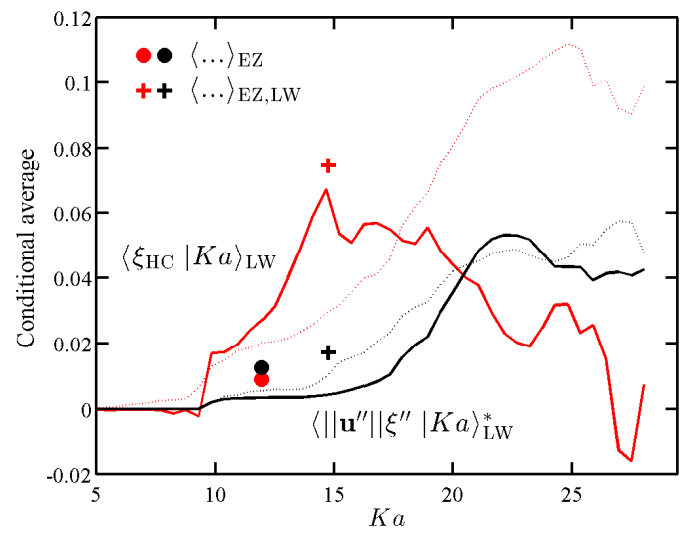

(c)

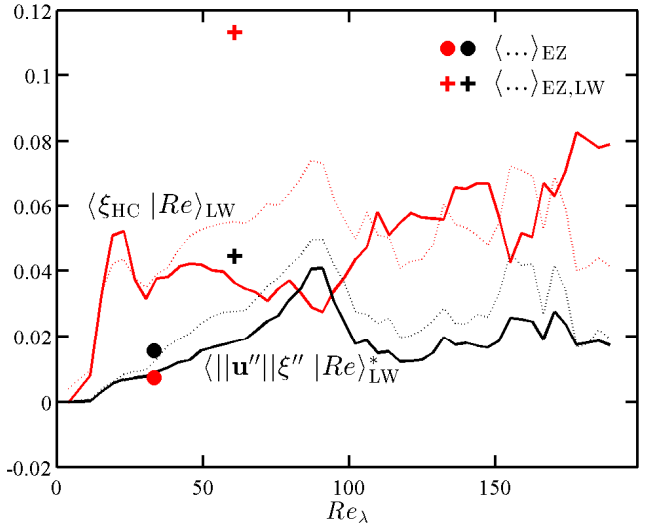

(b)

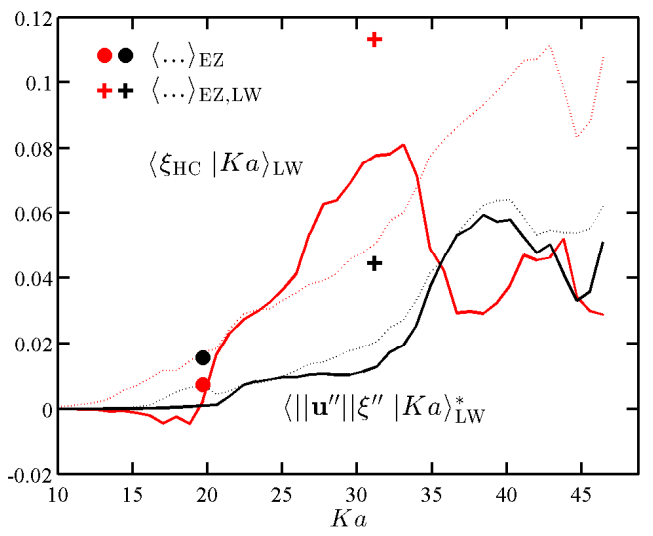

(d)

Figure 6: Conditional averages of $\left\langle\xi_{\mathrm{HC}} \mid R e_{\lambda}\right\rangle$ (red solid line) and $\left\langle\left\|\mathbf{u}^{\prime \prime}\right\| \xi^{\prime \prime *} \mid R e_{\lambda}\right\rangle$ (black solid line) and their standard deviation (dotted line) for the CO-lean (a) and the CO-rich (b) cases. Similar averages conditioned based on local Karlovitz number, $\left\langle\xi_{\mathrm{HC}} \mid K a\right\rangle$ (red solid line) and $\left\langle\| \mathbf{u}^{\prime \prime}|| \xi^{\prime \prime *} \mid K a\right\rangle$ (black solid line) are also shown for the CO-lean (c) and the CO-rich (d) cases. Volume averages constructed from explosive zones in the entire domain (EZ) and explosive zones in the leeward sampling region (EZ, LW) are also shown. The red circle denotes $\left\langle\xi_{\mathrm{HC}}\right\rangle_{\mathrm{EZ}}$, the black circle $\left\langle\left\|\mathbf{u}^{\prime \prime}\right\| \xi^{\prime \prime *}\right\rangle_{\mathrm{EZ}}$, the red plus $\left\langle\xi_{\mathrm{HC}}\right\rangle_{\mathrm{EZ}, \mathrm{LW}}$, the black plus $\left\langle\left\|\mathbf{u}^{\prime \prime}\right\| \xi^{\prime \prime *}\right\rangle_{\mathrm{EZ}, \mathrm{LW}}$. 


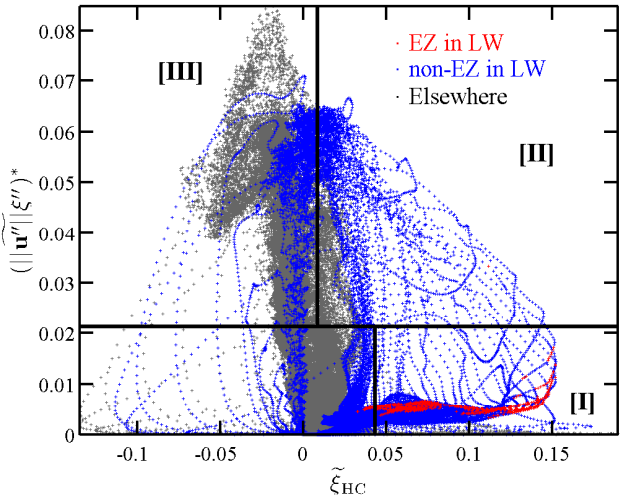

(a)

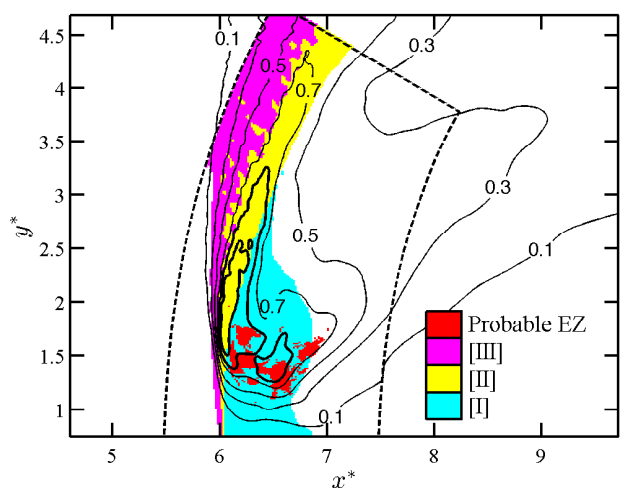

(c)

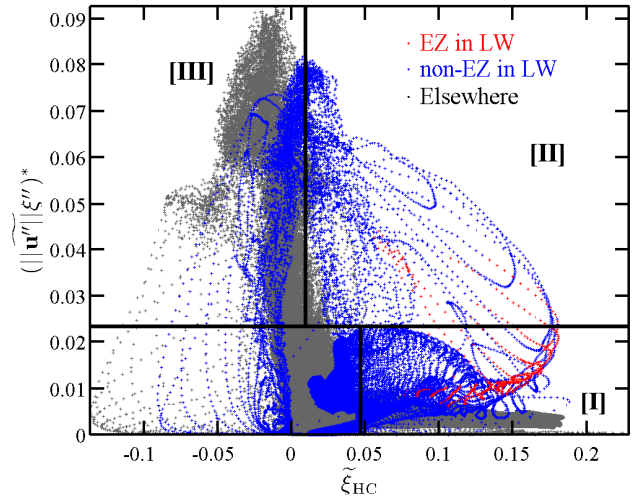

(b)

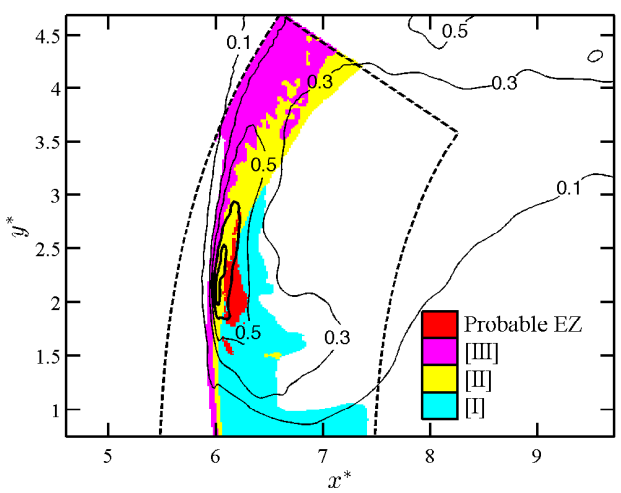

(d)

Figure 7: Variation of $\| \widetilde{\mathbf{u}^{\prime \prime} \| \xi^{\prime \prime}}{ }^{*}$ with $\widetilde{\xi}_{\mathrm{HC}}$ with the classification of zones [1]-[3] (a, b), and a map showing these zones in the LW region in physical space $(c, d)$ for the CO-lean $(\mathrm{a}, \mathrm{c})$ and the CO-rich $(\mathrm{b}, \mathrm{d})$ cases. In $(\mathrm{a}, \mathrm{b})$, red: samples taken from the probable EZ $\left(p_{E Z}^{\circ}>0.5\right)$ in the LW sampling region, blue: non-EZ samples in the LW sampling region, and gray: other samples in entire domain. In (c, d), iso-lines of $\bar{Q}^{\circ}=0.1,0.3,0.5,0.7$ (thin lines), 0.8, 0.9 (bold line) for the CO-lean and $\bar{Q}^{\circ}=0.1,0.3,0.5$ (thin lines), 0.7, 0.8 (bold line) for the CO-rich cases. Zone [I]: Large $\widetilde{\xi}_{\mathrm{HC}}^{\circ} \geq 0.25$ and negligible $\widetilde{\left\|\mathbf{u}^{\prime \prime}\right\| \xi} \stackrel{\circ}{\circ} \leq .25$, zone $[\mathrm{II}]: \widetilde{\xi}_{\mathrm{HC}}^{\circ} \geq 0.05$ and large $\| \widetilde{\mathbf{u}^{\prime \prime} \| \xi^{\prime \prime}}>0.25$, and zone [III]: negative or negligible $\widetilde{\xi}_{\mathrm{HC}}^{\circ}<0.05$ and large $\| \widetilde{\mathbf{u}^{\prime \prime} \| \xi^{\prime \prime}} \geq 0.25$. 


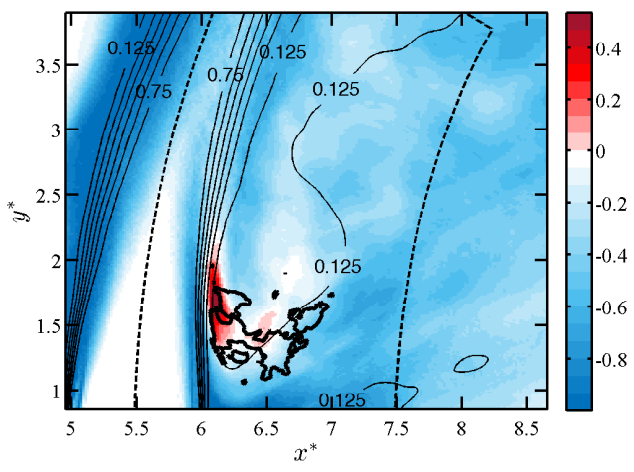

(a)

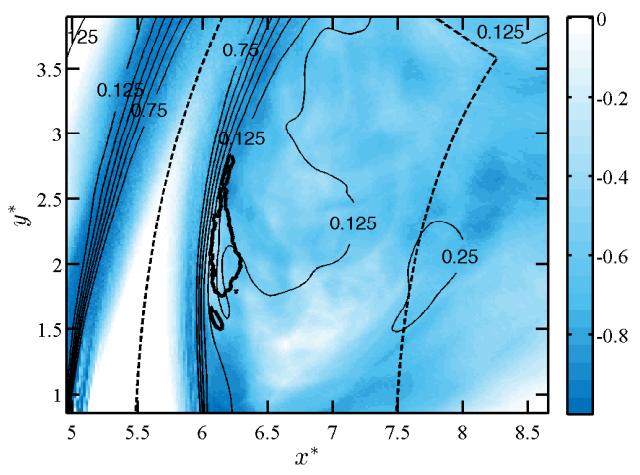

(b)

Figure 8: Iso-lines of mean velocity magnitude $\|\widetilde{\mathbf{u}}\|^{*}=0.125,0.25, \cdots, 0.75$ (thin solid line) and probable explosive zones $p_{E Z}^{\circ}=0.5$ (bold solid line) overlaid on color iso-contours of the mean flame index $\overline{\mathrm{FI}}$ for the CO-lean (a) and the CO-rich (b) cases. 


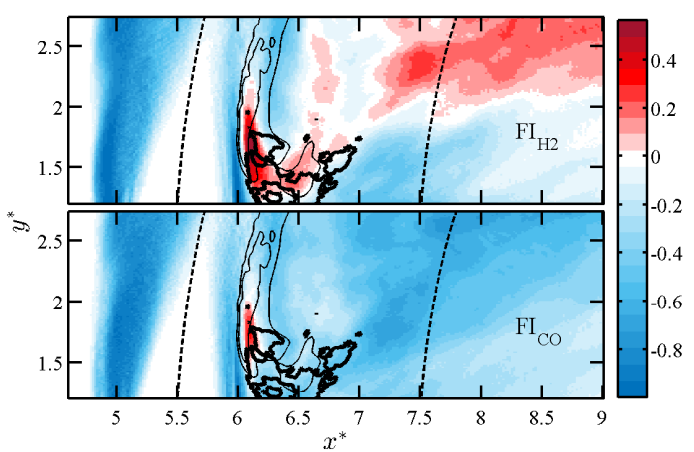

(a)

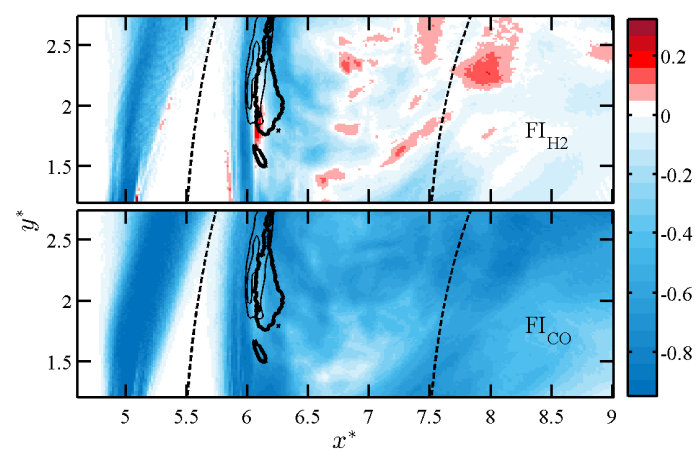

(b)

Figure 9: Iso-lines of the mean heat release rate $\bar{Q}^{\circ}=0.8,0.9$ (a) and $\bar{Q}^{\circ}=0.7,0.8$ (b) (thin solid line) and the probable explosive zones $p_{E Z}^{\circ}=0.5$ (bold solid line) overlaid on color iso-contours of the mean Flame Indices $\overline{\mathrm{FI}}_{\mathrm{H}_{2}}$ (top) and $\overline{\mathrm{FI}}_{\mathrm{CO}}$ (bottom) for the CO-lean (a) and the CO-rich (b) cases. 
Table 1: Ensemble averages in the explosive zones (EZ) in the LW, WW and other sampling locations.

\begin{tabular}{cccc} 
CO-lean/rich & EZ $(\mathrm{LW})$ & EZ $(\mathrm{WW})$ & EZ (elsewhere) \\
\hline$\langle\xi\rangle$ & $0.16 / 0.27$ & $0.35 / 0.43$ & $0.18 / 0.26$ \\
$\langle N\rangle^{*}$ & $4.0 \times 10^{-4} / 2.3 \times 10^{-3}$ & $4.6 \times 10^{-3} / 4.5 \times 10^{-3}$ & $3.4 \times 10^{-4} / 5.8 \times 10^{-4}$ \\
$\langle F I\rangle$ & $0.37 /-0.17$ & $-0.42 /-0.75$ & $-0.17 / 0.026$ \\
$\left\langle F I_{\mathrm{H}_{2}}\right\rangle$ & $0.60 / 0.19$ & $-0.37 /-0.58$ & $0.13 / 0.38$ \\
$\left\langle F I_{\mathrm{CO}}\right\rangle$ & $-0.13 /-0.20$ & $-0.45 /-0.74$ & $-0.38 /-0.0039$ \\
$\left\langle\xi_{\mathrm{HC}}\right\rangle$ & $0.075 / 0.11$ & $0.094 / 0.17$ & $-0.0063 / 8.0 \times 10^{-4}$ \\
$\left\langle\left\|\mathbf{u}^{\prime \prime}\right\| \xi^{\prime \prime}\right\rangle^{*}$ & $0.017 / 0.044$ & $0.064 / 0.17$ & $0.011 / 0.014$ \\
$\langle Q\rangle / \bar{Q}_{\max }$ & $1.4 / 1.1$ & $0.93 / 1.2$ & $0.95 / 0.90$
\end{tabular}

